\title{
Influence of family environment on the outcomes of cochlear implantation in pediatric recipients
}

Saad H. Alenzi, MD, Roa T. Halawani, MD, Afrah M. Alshalan, MD, Sara A. Habis, MD, Abdulrahman A. Alsanosi, MD.

\begin{abstract}

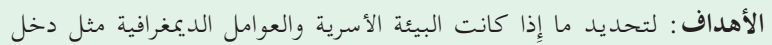

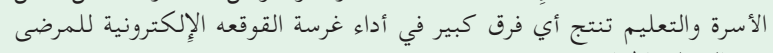
بعد العملية الجراحية.

المنهجية: في هذه الدراسة ، تم تضمين 49 مشاركًا من زارعي أجهزة

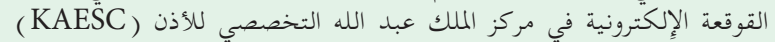

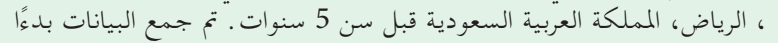

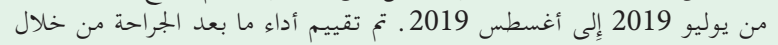

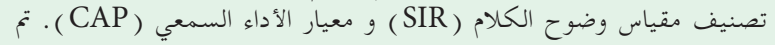

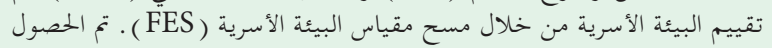

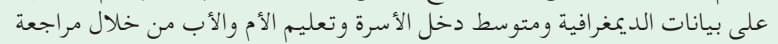

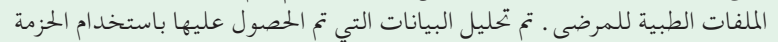

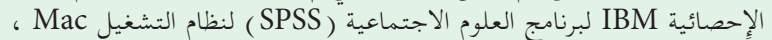
الإصدار 23.

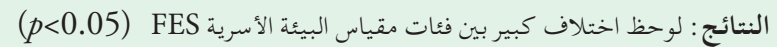
من حيث درجات CAP بعد العملية ، بينما كان هنان كناك تأثير كبير للفئة التعبيرية

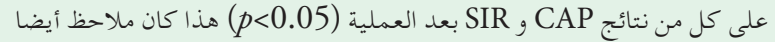

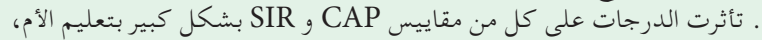
وكانت الدرجات على مقياس SIR مرتبطة بشكل إيجابي بتعليم الأب بـ كليم

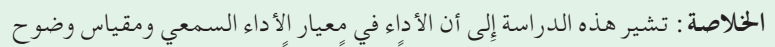
الكلام في مرضى زارعي القوقعة مرتبطاً ارتباطًا وثيقًا بالبيئة الأسرية بالإضافة إلى إلى

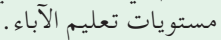

Objectives: To determine whether family environment and demographic factors such as family income and education produce any significant difference in postoperative performance of cochlear implant (CI) patients.

Methods: In this study, 49 participants who received cochlear implant devices at King Abdullah Ear Specialist Center, Riyadh, Saudi Arabia before the age 5 years were included. Data were collected between July 2019 and August 2019. Postoperative performance was assessed by speech intelligibility rating (SIR) and categories of auditory performance (CAP) scales. Family environment was assessed with family environment scale (FES) survey. Data of demography, average income, and maternal and paternal education were obtained through review of patient's medical files. The data obtained were analyzed using Statistical Package for Social Sciences for Mac, version 23 (IBM Corp, Armonk, NY, USA).

Results: A significant difference between the organization categories of FES $(p<0.05)$ was observed in terms of postoperative CAP scores, while a significant effect of the expressiveness category on both postoperative CAP and SIR scores $(p<0.05)$ was observed. The scores on both CAP and SIR scales were significantly affected by maternal education, and the scores on SIR scale were positively correlated with paternal education.

Conclusion: This study suggests that post-procedural performance of auditory perception and speech intelligibility in CI patients is significantly related to the family environment as well as the education levels of the parents.

Keywords: categories of auditory performance, speech intelligibility rating, cochlear implant

Saudi Med J 2020; Vol. 41 (5): 485-490 doi: 10.15537/smj.2020.5.25070

From the Department of Otolaryngology-Neurotology \& Skull Base Surgery (Alenzi), King Abdullah Ear Specialist Center; from the Department of Otolaryngology-Neurotology \& Skull Base Surgery (Alenzi, Alsanosi), from the Department of Otolaryngology-Neurotology \& Skull Base Surgery (Alshalan), from the Department of Medicine (Habis), King Saud University, Riyadh; and from the Department of Otolaryngology-Neurotology \& Skull Base Surgery (Halawani), Ohuhd Hospital Ministry of Health, Al Madinah Al Munawarah, Kingdom of Saudi Arabia.

Received 10th December 2019. Accepted 9th April 2020.

Address correspondence and reprint request to: Dr. Saad H. Alenzi, Department of Otolaryngology-Neurotology \& Skull Base Surgery, King Saud University, Riyadh, Kingdom of Saudi Arabia.E-mail: ent7052@gmail.com

ORCID ID: https://orcid.org/0000-0003-2556-5043 
$\mathrm{M}$ odern cochlear implants (CI) was first introduced in 1977 , are now the procedure of choice in severe to profound sensorineural hearing loss that does not benefit from standard hearing aids. Cochlear implants improves speech perception and working memory when implanted bilaterally in children, ${ }^{1}$ which leads to positive impact on the confidence, daily and social activities, and overall psychological status of the patients.

Research has revealed the various benefits of implantation of CI devices, particularly an improvement of exposure to spoken communication that enables personal development of language and speech skills in children. Based on this phenomenon, many studies have focused on the positive impacts of early implantation which is considered as an index procedure in children with hearing disability between the ages of 15 months to 3 years. In majority of these studies, the results revealed that early procedure alleviates the quality of life (QoL) index of the patient with better speech production scores, auditory performance, leading to more chances of these patients getting mainstream education. ${ }^{2,3}$ Necula et al, ${ }^{4}$ reported that health-related QoL was positively correlated with speech intelligibility and auditory performance, and negatively correlated with age at implantation, and that CIs were effective in improving the socializing abilities of hearing-impaired children with their normal-hearing peers.

The performance of CI should be carefully assessed to determine the success rate of operation and long-term outcome, since children undergoing the same intervention may achieve different levels of benefit due to individual differences. Various tools are available to assess the performance of CIs during follow-up visits. Specially, categories of auditory performance (CAP) scale is an index of 8 categories to determine outcomes of CI procedures in daily life; the categories in CAP are in order of increasing difficulty, but can be easily understood and applied by parents and non-specialist professionals. ${ }^{5}$ The categories in CAP scale range from 0 to 7 with category 0 meaning that the patient has no awareness of sounds around them and category 7 meaning that the patient is able to use the telephone. Speech intelligibility rating (SIR) scale is another tool to assess CI performance, which both parents and

Disclosure. Authors have no conflict of interests, and the work was not supported or funded by any drug company. non-specialist professionals can administer with ease. ${ }^{6}$ Speech intelligibility rating scale is based on 5 categories of speech intelligibility, with category one meaning that the major part of a patient's communication is manual and category 5 meaning that patient's speech is understood easily. Both these scales are valid and reliable to assess speech production and auditory perception of CI children. ${ }^{7}$

Research has focused on factors that contribute to the success of CI, which raised the question whether family environment is a direct influencing factor of cognition, behavior and social development in children with CI. Since subjects who have undergone $\mathrm{CI}$ procedure may experience social and attention problems that can increase parental stress, good family support, and aural-verbal rehabilitation are particularly important for improvement of behavioral outcomes in children with CI. ${ }^{8}$

The family environment scale (FES) has been used to assess the impact of family on the outcome of CIs in children; FES is a 90-item self-report true-false questionnaire that assesses 3 dimensions of family environment: a) interpersonal relationships within the family (relationship), b) goals, activities, and interests within the family (personal growth), and c) structure, organization, and rules in running the family (system maintenance), using 10 subscales. ${ }^{9}$ The respondents of a family answer individually and decide which statement is true or false for his or her family. The question statements are fairly simple and easily comprehensible irrespective of the level of education of the respondent. Expressiveness, cohesion, and conflict are used to evaluate interpersonal relationships. Moralreligious orientation, achievement orientation, activerecreational orientation, and independence subscales evaluate personal growth. System maintenance is measured by control and organization. Each category is scored on a scale of 0 to 9 , with 9 representing the strongest perceptions about that specific category. According to the instruction from the manual, these raw scores are then converted into a standard score. Higher scores represent higher perceptions for that specific category. The normal limits for any category are 40 to 60 . The categories in which the score is above 60 represent strong perceptions of the respondent in those categories and the ones where the score is less than 40 represent weak perceptions. The FES real form (form $\mathrm{R}$ ) comprises items related to parent's view of family status, as well as view-point of ideal status of the immediate family, and is usually filled by both parents.

This study would try to find correlations between CAP and SIR scores and family environment and prove if 
any specific family factors or other demographic factors are linked with better outcomes after CI procedures.

Methods. This cross-sectional study was conducted at King Abdullah Ear Specialist Center (KAESC), King Abdulaziz University Hospital, Riyadh, Saudi Arabia. The inclusion criteria were both male and female children who received CI at KAESC between 2013 to 2016, and those using CI for $>3$ years. Based on the fact that all assessment tools are based on speech comprehension, non-Arabic speakers were excluded from the study; in addition, patients with postoperative device failure, and those with other health problems that affect mental status such as autism and motor neuron disease (MND) were excluded.

Forty-nine patients who were given CI between 2016 and 2019 were included and data were collected by following up on them between July 2019 and August 2019. After obtaining consent, patient data were collected by review of the medical charts; performance of CI was assessed by 2 assessment tools, SIR and CAP, at regular follow-up visits, and the results were documented in the child's medical file at KAESC. Subsequently, socioeconomic survey by phone interview, and FES, a validated scale for social environment of the family unit, were conducted in each patient. The interview was conducted in Arabic language. Copyrights to FES scale along with permission to translate into Arabic were acquired from the relevant parties. The study was approved by the Institutional Review Board at King Saud University, Riyadh, Saudi Arabia (Research and Development agreement and ethical approval No. 17/0860/IRB).

Statistical analysis. Information of different demography factors such as age, mother and father's education, and family income was obtained from the medical files. Pearson's correlation and 2-tailed significance tests were used to find out correlations. The data obtained were analyzed using Statistical Package for Social Sciences for Mac, version 23 (IBM Corp, Armonk, NY, USA).

Results. A total of 49 subjects were included in the study, of which, 21 were male and 28 were female. The subjects' ages were in the range of 5 to 13 years (average age, 8.26 years). In all patients, surgery for cochlear-device implantation was performed between 1 to 5 years of age (average age, 3.48 years) (Table 1). Five $(10.2 \%)$ families had the monthly income of less than 5000 Saudi Riyals (SR), 34 (69.4\%) families between 5000 and 10000 SR, and 10 (20.4\%) families between 10000 and 20000 SR. Five (10.2\%) patients had fathers with only middle school education, 13 (26.5\%) high school, 27 (55.1\%) had Bachelors degree, and only 4 $(8.2 \%)$ had acquired masters or higher degrees. On the other hand, $2(4.1 \%)$ mothers had no formal education at all, 1 (2\%) was primary school graduate, 7 (14.3\%) were middle school graduates, 15 (30.6\%) were high school graduates, and 24 (49\%) had Bachelors degree.

The mean \pm SD value for CAP was $6.63 \pm 2.05$, and that for SIR was $2.95 \pm 1.44$. It should be noted that higher scores of CAP and SIR scales indicate better outcomes. In FES, highest scores (average, 57) were obtained in the control category, and lowest (average, 38.12) were obtained in the intellectual-cultural orientation category. Two subscales (intellectualcultural orientation and active-recreational orientation) were less than the lower limit of 40 . Detailed results are shown in Table 2.

Among variables other than FES subscales, the mothers' level of education was found to have a significant correlation with both CAP $(p=0.045)$ and SIR $(p=0.013)$ variables. The father's education was

Table 1 - Age at the time of study and at surgery.

\begin{tabular}{lcc}
\hline Variables & Age of the patient & $\begin{array}{c}\text { Age at the time of } \\
\text { surgery }\end{array}$ \\
\hline Number of subjects & 49 & 49 \\
Mean & 8.270 & 3.480 \\
Standard deviation & 1.810 & 1.800 \\
Minimum & 5.000 & 1.000 \\
Maximum & 13.000 & 5.000 \\
\hline
\end{tabular}

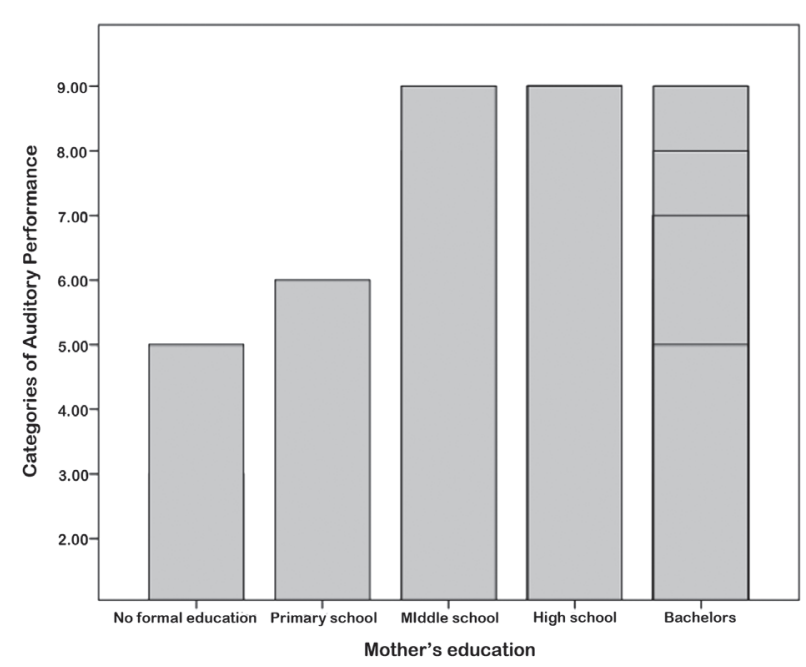

Figure 1 - Categories of auditory performance scores and mother's education. 
significantly correlated with only the SIR $(p=0.036)$ variable. The rest of the demographic including age of the patient, surgery age (under 5 years), and income were found to be insignificant in determining the outcome of CI procedures. Details are shown in Table 3. The relationship between CAP and SIR

Table 2 - Family environment scale (FES) scores.

\begin{tabular}{lccccc}
\hline FES Subscales & $\begin{array}{c}\text { Number of } \\
\text { subjects }\end{array}$ & Minimum & Maximum & Mean & $\begin{array}{c}\text { Standard } \\
\text { deviation }\end{array}$ \\
\hline Cohesion & 49 & 25 & 65 & 52.760 & 10.507 \\
Expressiveness & 49 & 22 & 59 & 43.940 & 9.404 \\
Conflict & 49 & 33 & 80 & 48.490 & 10.953 \\
Independence & 49 & 13 & 61 & 40.730 & 12.548 \\
$\begin{array}{l}\text { Achievement orientation } \\
\text { Intellectual-cultural }\end{array}$ & 49 & 16 & 72 & 52.530 & 12.440 \\
orientation & 49 & 19 & 63 & 38.120 & 10.325 \\
$\begin{array}{l}\text { Active-recreational } \\
\text { orientation }\end{array}$ & 49 & 28 & 64 & 38.430 & 9.781 \\
Moral-religious emphasis & 49 & 36 & 66 & 54.370 & 7.245 \\
Organization & 49 & 26 & 69 & 50.160 & 11.421 \\
Control & 49 & 38 & 76 & 57.000 & 10.336 \\
\hline
\end{tabular}

Table 3 - Pearson correlation test between categories of auditory performance (CAP), speech intelligibility rating (SIR), and the other demographic variables.

\begin{tabular}{lcccc}
\hline Variables & \multicolumn{2}{c}{ CAP } & \multicolumn{2}{c}{ SIR } \\
& Pearson correlation & $P$-value & Pearson correlation & $P$-value \\
\hline Age of the patient & 0.114 & 0.312 & 0.159 & 0.201 \\
Age at the time of surgery & 0.066 & 0.621 & 0.095 & 0.376 \\
Monthly income & 0.235 & 0.251 & 0.174 & 0.320 \\
Father's education & 0.266 & 0.124 & 0.305 & 0.036 \\
Mother's education & 0.294 & 0.045 & 0.401 & 0.013 \\
\hline
\end{tabular}

Table 4 - Pearson correlation test between categories of auditory performance (CAP), speech intelligibility rating (SIR), and family environment scale (FES) subscales.

\begin{tabular}{lcccc}
\hline FES subscales & \multicolumn{2}{c}{ CAP } & \multicolumn{2}{c}{ SIR } \\
& Pearson correlation & $P$-value & Pearson correlation & $P$-value \\
\hline Cohesion & 0.032 & 0.825 & -0.120 & 0.411 \\
Expressiveness & -0.287 & 0.045 & -0.350 & 0.014 \\
Conflict & -0.093 & 0.523 & -0.033 & 0.822 \\
Independence & 0.174 & 0.231 & 0.040 & 0.787 \\
Achievement orientation & 0.280 & 0.051 & 0.192 & 0.187 \\
Intellectual-cultural orientation & 0.079 & 0.591 & -0.009 & 0.949 \\
Active-recreational orientation & 0.134 & 0.358 & 0.180 & 0.216 \\
Moral-religious emphasis & 0.057 & 0.699 & 0.093 & 0.524 \\
Organization & 0.297 & 0.038 & 0.198 & 0.173 \\
Control & 0.138 & 0.344 & 0.082 & 0.573 \\
\hline
\end{tabular}




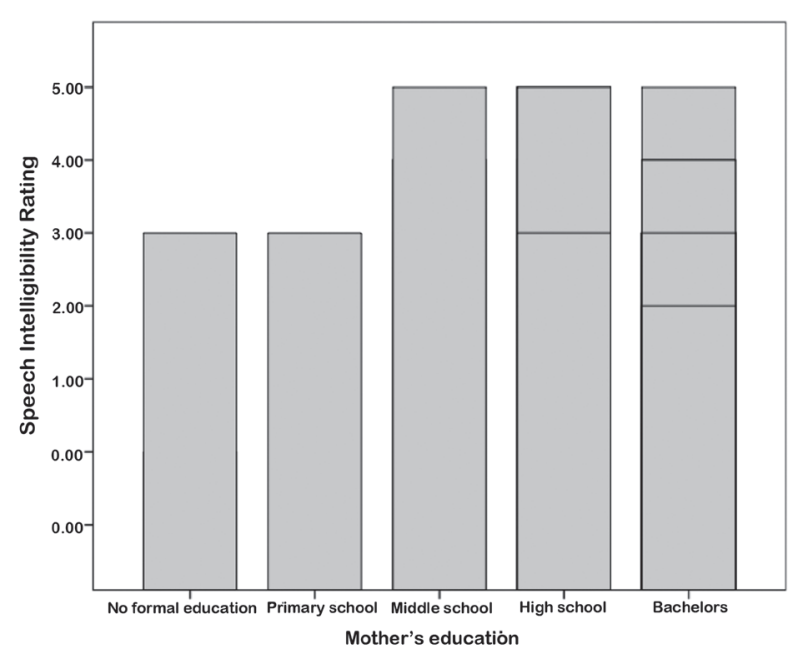

Figure 2 - Speech intelligibility rating scores and mother's education

variables and mother's education level is shown in Figures $1 \& 2$. It is shown that higher education is positively correlated with better CAP and SIR scores. The relationship between SIR scores and father's education is given in Figure 3.

Using Pearson's correlation, it was found that expressiveness was significantly related with both CAP and SIR $(p<0.05)$. Similarly, significant relationships were observed between the category of organization and CAP $(p<0.05)$ when Pearson's correlation test was applied. Relationships between the other variables and SIR are shown in Table 4.

Discussion. Our study highlighted the impact of family environment and other demographic variables on postoperative performance of $\mathrm{CI}$ in children undergoing implantation surgery before the age of 5 years. The patients were asked to complete the FES survey, the categories of which were then correlated with CAP and SIR scores.

In FES subscales, intellectual-cultural orientation and active-recreational orientation were found to be less than the normal range of 40 to 60 . Some of the categories did exceed the average of 50, but none crossed the upper limit of 60 . However, a study conducted on the family environment of pediatric CI patients found that the organization and cohesion mean values exceeded the normal values. ${ }^{10}$

Coming to the correlations, the results revealed that the CAP scale was positively correlated with expressiveness and organization. The scores on the SIR scale showed a significant relationship with the Expressiveness category. According to Moos and Moos, ${ }^{9}$

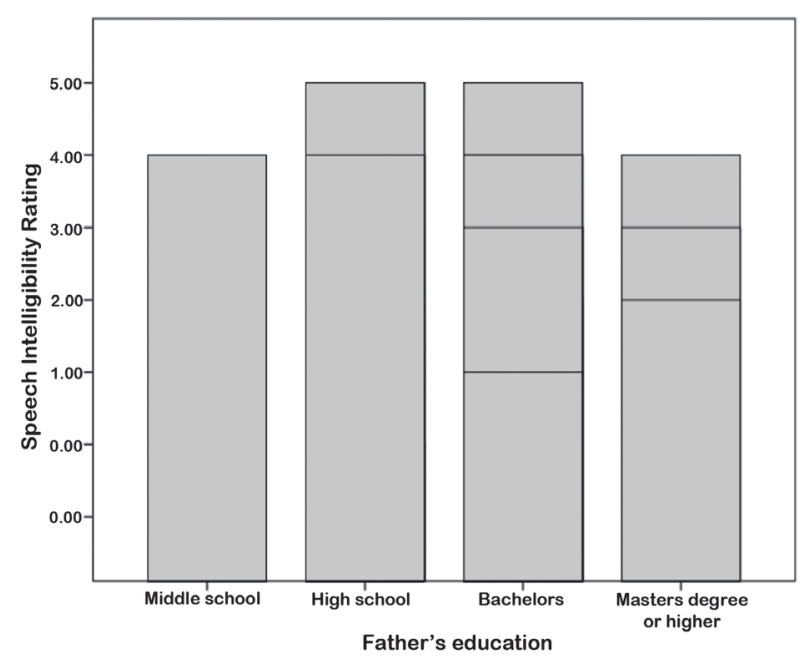

Figure 3 - Speech intelligibility rating scores and father's education.

organization measures the importance of order and organization in the family in terms of structuring the family activities, financial planning, and explicitness of attitudes to rules and responsibility, while expressiveness is defined as the extent to which family members are allowed and encouraged to act openly and express their feelings directly. On a broader scale, expressiveness is part of the personal growth category and organization is included in system maintenance according to the authors of the FES tool. Regarding correlations of SIR and CAP with FES, our findings are contrary to the results of the study carried out by Necula et $\mathrm{al}^{10}$ which found no significant relationships between FES subscales and these speech intelligibility and auditory perception scores.

The results of the present study also indicated a significant relationship between the scores obtained with CAP and SIR and the level of mother's education; in addition, the SIR scale showed positive correlation with the level of father's education. These findings can be explained by the fact that parental guidance plays a major role in the cognitive development of children, and good education background enables parents to meet the needs of children with hearing disability. In agreement, a report indicated that the mother's education level was directly related to the domain of socialization and communication in children. ${ }^{11}$

A study reported that a better socioeconomic environment results in significantly higher SIR scores in pediatric patients with CIs. ${ }^{12}$ However, our study did not produce similar results. The same study found no significant relationship between parents' level of education and SIR and CAP scores which is also in contrast to the findings of this study. 
Study limitations. This study include a small sample-size of 49 subjects and the findings may not be applicable in the general population. In an ideal case, a much larger sample is needed to develop hard proof of such correlations. Similarly, the scales used to evaluate auditory perception and speech intelligibility do not allow a complete analysis of each and every aspect of speech perception and intelligibility. This can explain the lack of more correlations between the family environment and post-procedural performance of patients.

In conclusion, factors related to family environment are linked to cognitive development in childhood, and under a better environment, they increase the chances of normal growth. Our study proves that there are significant correlations between certain family traits and post-procedural performance of CI patients. However, further studies are needed to develop indisputable proof of these correlation, so the family environment can be altered appropriately, in order to achieve the best possible results.

Acknowledgment. The authors would like to thank Dr. Yassin Abdelsamad, for his overall support in this study. The authors gratefully acknowledge Editage (www.editage.com) for English language editing.

\section{References}

1. Rayes H, Al-Malky G, Vickers D. Systematic review of auditory training in pediatric cochlear implant recipients. J Speech Lang Hear Res 2019; 62: 1574-1593.
2. le Roux T, Vinck B, Butler I, Cass N, Louw L, Nauta L, et al. Predictors of pediatric cochlear implantation outcomes in South Africa. Int J Pediatr Otorhinolaryngol 2016; 84: 61-70.

3. Tobey EA, Thal D, Niparko JK, Eisenberg LS, Quittner AL, Wang NY, et al. Influence of implantation age on school-age language performance in pediatric cochlear implant users. Int J Audiol 2013; 52: 219-229.

4. Necula V, Cosgarea M, Necula SE. Health-related quality of life in cochlear implanted patients in Romania. Int J Pediatr Otorhinolaryngol 2013; 77: 216-222.

5. Archbold S, Lutman ME, Marshall DH. Categories of auditory performance. Ann Otol Rhinol Laryngol Suppl 1995; 166: 312-314.

6. Cox RM, McDaniel DM. Development of the speech intelligibility rating (SIR) test for hearing aid comparisons. $J$ Speech Hear Res 1989; 32: 347-352.

7. Hassanzadeh S. The psychometric properties of the Persian version of categorization of auditory performance II and speech intelligibility rating scales in cochlear-implanted deaf children. Audiol 2015; 23: 76-84.

8. Chao WC, Lee LA, Liu TC, Tsou YT, Chan KC, Wu CM. Behavior problems in children with cochlear implants. Int J Pediatr Otorhinolaryngol 2015; 79: 648-653.

9. Maino E, Lanz M. Family environment scale. In: Michalos A.C, editors. Encyclopedia of quality of life and well-being research. Springer: Dordrecht; 2014.

10. Necula V, Cosgarea M, Maniu AA. Effects of family environment features on cochlear-implanted children. Eur Arch Otorhinolaryngol 2018; 275: 2209-2217.

11. DesJardin JL, Eisenberg LS. Maternal contributions: supporting language development in young children with cochlear implants. Ear Hear 2007; 28: 456-469.

12. Sharma S, Bhatia K, Singh S, Lahiri AK, Aggarwal A. Impact of socioeconomic factors on paediatric cochlear implant outcomes. Int J Pediatr Otorhinolaryngol 2017; 102: 90-97. 